\title{
ÜBERSICHTEN.
}

\section{EINIGE KAPITEL AUS DER PATHOLOGIE DER FRÜHGEBORENEN KINDER.}

\section{Von}

Prof. Dr. ARvo Ylppö, Helsingfors.

Unter einem frühgeborenen Kind hat man im allgemeinen bis jetzt ein solches verstanden, welches vor Ablauf einer normalen Schwangerschaft zur Welt gekommen ist. Nun wissen wir aber, daß die Angaben über die Daver der Schwangerschaft häufig sehr unsicher sind und auch in denjenigen Fällen, wo man genauer die Zeit der letzten Menstruation anzugeben vermag, sind wir im Ungewissen, wann eigentlich die Konzeption stattgefunden hat. Auf diese Weise können anamnestische Fehler von I-2 Monaten in bezug auf das Alter der Frucht entstehen.

Um nun eine sicherere, objektive und einheitlichere Definition für den Begriff ,,frühgeborenes Kind" zu erhalten, hat der Verfasser vorgeschlagen, daß man alle Kinder mit einem Geburtsgewicht unter $25^{\circ 0} \mathrm{~g}$ einfach als Frühgeburten betrachtet.

Die klinische Erfahrung zeigt nämlich, daß die Mehrzahl derjenigen Kinder, welche mit einem kleineren Körpergewicht als $2500 \mathrm{~g}$ geboren wurden, in bezug auf Pflege, Lebensfähigkeit und Körperfunktionen nicht als reif angesehen werden dürfen. Jedoch muß man zugeben, daß ausnahmsweise auch nach einer normalen Schwangerschaft, welche mindestens 280 Tage gedauert hat, Kinder mit einem Geburtsgewicht unter $2500 \mathrm{~g}$ geboren werden können. Das Wachstum dieser letzteren hat sich aus irgendeiner, sei es mehr exogen oder mehr endogen konstitutionellen Ursache verzögert. Während des intrauterinen Wachstums können wir unbeachtet der scheinbar gleichen Lebens- und Ernährungsbedingungen der Frucht doch große Differenzen bei den einzelnen Kindern vorfinden, genau so wie im extrauterinen Leben. Zum Beispiel können wir unter rojährigen Kindern Individuen treffen, die $\mathrm{I}_{5} \mathrm{~cm}$ und andere, die nur $125 \mathrm{~cm}$ lang sind, und alle beide können doch am Ende der Wachstumsperiode wieder gleich groß sein. So kann auch eine 8 Monate alte Frucht ausnahmsweise über $3000 \mathrm{~g}$ wiegen, eine andere dagegen weit unter $2000 \mathrm{~g}$. Hiermit ist aber nicht gesagt, daß die kleinere Frucht auch definitiv kleiner zu bleiben braucht.

Außerdem kennen wir eine Reihe von pathologischen Wachstumsstörungen wie die Chondrodystrophie und andere hypoplastische Zustände, bei welchen bereits während des intrauterinen Lebens das Wachstum deutlich gehemmt wurde. Ziehen wir hier nur diejenigen Kinder, die unter $2500 \mathrm{~g}$ bei der Geburt wiegen, in Betracht, so ist es doch erstaunlich, daß in Deutschland durchschnittlich $5 \%$ aller Kinder als Frühgeburten zur Welt kommen.

Bei einzelnen Autoren findet man sogar noch größere Zahlen. Nach einer großen französischen Sammelstatistik aus der "Maternité" und der BAUDELoq schen Klinik in Paris betrug die Anzahl der Frühgeburten sogar I 5,4\%. Diese Zahlen genügen schon, um zu zeigen, wie häufig die Frühgeburten sind und, wenn wir noch daran denken, daß es gerade die Frühgeborenen sind, die ganz besonders den Arzt in Anspruch nehmen, so ergibt sich ohne weiteres, daß die Kenntnisse der verschiedenen klinisch-pathologischen Erscheinungen bei diesen Kindern besonders wichtig für die ärztliche Praxis sind.

Wenn ich auch noch erwähne, daß unter den kranken Säuglingen in den Kinderkliniken die Zahl der Frühgeburten $20-25 \%$ beträgt, das heißt, daB jedes 4 . bis 5 . Kind eigentlich eine Frühgeburt ist, so ist ohne weiteres klar, daß unter den Kinderkrankheiten gerade die der frühzeitig geborenen Kinder eine sehr große Rolle spielen.

Die Anzahl der am Leben bleibenden Frühgeburten ist aber nun lange nicht so gro $B$ wie die der Geborenen überhaupt, denn die Sterblichkeit ist unter diesen eine auffallend große. Nach verschiedenen Statistiken schwankt sie außerordentlich je nach dem Geburtsgewicht. Die Lebensfähigkeit ist.in der Regel um so geringer je kleiner die Frucht bei der Geburt war. Die kleinsten Kinder, die überhaupt am Leben bleiben können, müssen mindestens $75^{\circ} \mathrm{g}$ wiegen (D'OUTREPOINT, OBERWART), bei noch kleineren ist jede auch noch so große Mühe umsonst.

Das Alter dieser kleinsten Früchte, die am Leben bleiben können, schwankt nach der Erfahrung des Verfassers zwischen

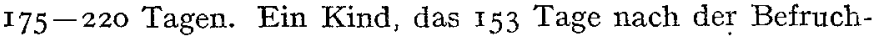
tung mit Io50 $\mathrm{g}$ Geburtsgewicht und einer Länge von $37 \mathrm{~cm}$ zur Welt kam, lebte auch sogar I4 Tage. Die Konzeption dieses Kindes konnte deshalb auf einen ganz bestimmten Tag festgesetzt werden, weil an dem Tage der Vater des Kindes aus dem Felde auf der Durchreise zu. Hause gewesen war, und im betreffenden Fall wir bei der jungen Frau auch allen Grund hatten, an die eheliche Treue zu glauben.

Nach dem deutschen bürgerlichen Gesetzbuch § I7I7, Abs. 2; heißt es, daß als Empfängniszeit die Zeit von I $8 \mathrm{r}$ bis 302 Tage von der Geburt des Kindes rückwärts gerechnet gilt. Diese untere Grenze für lebendgeborene Kinder liegt demnach viel $z \mathfrak{u}$ hoch und die betreffenden Paragraphen müßten abgeändert werden. Von verschiedenen Autoren (ZANGEMEISTER $u$. a.) ist bereits früher betont, daß 3 die obere Grenze, 302 Tage, für die Schwangerschaftsdauer zu niedrig gezogen ist.

Wie groß die Sterblichkeit unter den Frühgeburten im I. Lebensjahre ist, geht aus der beigefügten Tabelle hervor.

\begin{tabular}{|c|c|c|c|c|c|c|}
\hline \multirow[b]{2}{*}{ Geburtsgewicht } & $\begin{array}{c}\text { Anzahl } \\
\text { der }\end{array}$ & \multicolumn{5}{|c|}{ Gestorben bis zum Alter von } \\
\hline & $\begin{array}{c}\text { beob- } \\
\text { achteten } \\
\text { Kinder }\end{array}$ & I Tag & 5 Tage & I Monat & 6 Monate & I Jahr \\
\hline $600-1000 \mathrm{~g}$ & 37 & $\begin{array}{c}14 \\
37,84 \%\end{array}$ & $\begin{array}{c}27= \\
72,84 \%\end{array}$ & $\begin{array}{c}31= \\
83,79 \%\end{array}$ & $\begin{array}{c}33= \\
89,19 \%\end{array}$ & $\begin{array}{c}34= \\
91,89 \%\end{array}$ \\
\hline $\mathrm{IOOI}-\mathrm{I} 500 \mathrm{~g}$ & 178 & $\begin{array}{c}28= \\
\mathrm{I} 5,73 \%\end{array}$ & $\begin{array}{c}57= \\
32,02 \%\end{array}$ & $\begin{array}{c}88= \\
49,43 \%\end{array}$ & $\begin{array}{l}\operatorname{I} x= \\
62,36 \%\end{array}$ & $\begin{array}{c}\text { I I } 4= \\
64,04 \%\end{array}$ \\
\hline $\mathrm{I} 5 \mathrm{OI}-2000 \mathrm{~g}$ & 233 & $\begin{array}{l}\text { I } 6= \\
6,87 \%\end{array}$ & $\begin{array}{c}26= \\
1,16 \%\end{array}$ & $\begin{array}{c}56= \\
24,03 \%\end{array}$ & $\begin{array}{c}8.3= \\
35,62 \%\end{array}$ & $\begin{array}{c}96= \\
4 \mathrm{I}, 20 \%\end{array}$ \\
\hline $200 \mathrm{I}-2500 \mathrm{~g}$ & $20 I$ & $\begin{array}{c}4= \\
1,99 \%\end{array}$ & $\begin{array}{l}10= \\
4,98 \%\end{array}$ & $\begin{array}{c}3 I= \\
15,42 \%\end{array}$ & $\begin{array}{c}48= \\
23.88 \%\end{array}$ & $\begin{array}{c}58= \\
28,86 \%\end{array}$ \\
\hline
\end{tabular}

Von diesen 649 Frühgeburten, die im , Kaiserin-AugusteVictoria-Haus" von Juni Igog bis Mai I9I8 geboren resp. wegen ihrer Unreife aufgenommen worden waren und deren Schicksal der Verfasser verfolgen konnte, sind im ganzen $53,5 \%$ gestorben. Die Sterblichkeit ist gerade in den ersten Lebenstagen besonders groß und, wie aus der Tabelle ersichtlich, unter den kleinsten am größten.

Beider Beurteilung der Lebensfähigkeit eines Frühgeborenen spielt die Ursache der vorzeitigen Geburt neben dem Geburtsgewicht eine große Rolle. In denjenigen Fällen, in welchen die Frühgeburt als Folge einer Infektionskrankheit der Mutter, wie Scharlach, Pneumonie, Variola, Sepsis, Lues usw. betrachtet werden $\mathrm{mu} B$, ebenso wie bei allen artifiziellen Geburten ist die Prognose bedeutend schlechter als bei spontanen aus unbekannter Ursache.

Unter diesen unbekannten Gründen spielen chronische Katarrhe in den Geburtswegen eine sehr große Rolle und sehr viele von denjenigen Frühgeburten, die von den Müttern auf irgendein angebliches, nicht genauer bekanntes Trauma 
zurückgeführt werden, stehen wohl mit einer chronischen Entzündung der Geburtswege im Zusammenhang. Insbesonders möchte ich hier auch betonen, daß die Lues keineswegs eine so große Rolle in der Ätiologie der Frühgeburten spielt, wie im allgemeinen angenommen wird. In meinem Material war nur in $5 \%$ der Fälle Lues klinisch oder serologisch nachweisbar, wobei ich erwähnen möchte, daß wir nicht nur in zweifelhaften und unklaren Fällen, sondern eine Zeitlang überhaupt bei jeder Frühgeburt die Wassermannsche Reaktion angestellt haben.

Verfolgen wir nun die weitere Entwickelung dieser Kinder nach der Geburt, so ist sie in erster Linie davon abhängig, wie groß das Geburtsgewicht war. Die verschiedenen ätiologischen Momente, mit Ausnahme von Lues und Tuberkulose, scheinen keine so große Rolle $\mathrm{zu}$ spielen wie das Geburtsgewicht und auch die Schädigung des Kindes unter der Geburt.

Wer eine größere Anzahl von Frühgeborenen nach der Geburt klinisch genauer untersucht hat, kann feststellen, daß bei denselben mit auffallender Häufigkeit mehr oder minder ausgedehnte Hämorrhagien in der Haut nachzuweisen sind. Besonders fällt in die Augen, daß im Bereich der Gesichts- und der Kopfhaut diese Hämorrhagien sehr ausgedehnt sind. Bei der Sektion von Frühgeburten kann man fernerhin nachweisen, da $B$ auch in den verschiedenen inneren Organen während der Geburt Blutungen sehr häufig auftreten.

Der Verfasser hat bei ca. 300 Frühgeburten, die in den ersten Lebenstagen resp. -monaten starben, mit erstaunlicher Regelmäßigkeit gerade bei den allerkleinsten Frühgeburten, die in den ersten Lebenstagen zugrunde gingen, ausgedehnte Hämorrhagien in den inneren Organen gefunden. In sehr vielen Fällen können diese Hämorrhagien, besonders in der Schädelhöhle, eine solche Ausdehnung annebmen, daß man sie als die direkte Todesursache betrachten muß. Aber sehr viele Frühgeburten mit geburtstraumatischen Blutungen in der Schädelhöhle und anderen inneren Organen bleiben doch am Leben und ihre Entwickelung bietet dann in den folgenden Lebensmonaten oder, -jahren vielerlei pathologische Erscheinungen, über deren Atiologie wir bis jetzt nicht so recht im klaren waren.

Häufigkeit der Gehirn- und Rückenmarksblutungen:

\begin{tabular}{l|c|c|c}
\hline Geburtsgewicht & $\begin{array}{c}\text { Anzahi der } \\
\text { Kinder } \\
\text { mit Schädel- } \\
\text { sektion }\end{array}$ & $\begin{array}{c}\mid c \\
\text { Davon Gehirn- und } \\
\text { Rückenmarksblutungen } \\
\text { a) Anzahl } \\
\text { der Fälle }\end{array}$ & b) \% \\
\hline \hline unter I000 g & 20 & I8 & 90 \\
100I-I500 g & $5 \mathrm{I}$ & 39 & 76,5 \\
I50I-2000 g & I7 & 6 & 35,3 \\
200I-2500 g & I5 & 4 & 26,7
\end{tabular}

Ich gebe hier eine auf Grund meines Sektionsmaterials aufgestellte Tabelle der Häufigkeit der Gehirn- und Rückenmarksblutungen wieder. Die Tabelle zeigt, daß tatsächlich bei den kleinsten Frühgeburten unter $1000 \mathrm{~g}$ Gewicht in $90 \%$ der Fälle Gehirn- oder, was ich auch hier besonders betonen möchte, Rückenmarksblutung bei der Sektion festgestellt werden konnten.

Fernerhin geht aus der Tabelle hervor, daß die Gehirnblutungen mit zunehmendem Geburtsgewicht seltener werden, daß sie also in engstem Konnex mit der Entwickelungsstufe des Kindes stehen. Nun finden wir bei den Frühgeburten im späteren Leben auffallend häufig verschiedene krankhafte Zustände, die auj Störungen im Zentralnervensystem deuten, vor allem verschiedene Intelligenzstörungen, von leichtesten auf weniger wichtigen geistigen Grebieten bis $\mathrm{zu}$ den schwersten Formen von Imbezillität und Idiotie. In $7-8 \%$ habe ich bei denjenigen Frühgeborenen; deren Schicksal ich eine längere Zeit habe verfolgen können, so schwergehende Intelligenzstörungen bei meinen Nachforschungen festgestellt, daß diese Kinder als Imbezille oder Idioten $\mathrm{zu}$ betrachten waren. Dazu kommt noch, daß der Litllesche Symptomenkomplex gerade bei den Frühgeburten mit be- sonderer Vorliebe und Häufigkeit auftritt. Ich habe Litcle in 3\% meiner Fälle nachweisen können.

Mit Litrie fasse ich hier nicht nur die typischen spastischen Hemi- oder Paraplegien auf, sondern überhaupt alle chronischen nicht funktionellen Spasmen in irgendeiner Muskelgruppe, sei es an den Extremitäten oder anderswo am Körper, überhaupt alle spastischen Nervenschädigungen, die scheinbar ohne näher bekannte Ursache langsam im Verlaufe des ersten Halbjahres aufgetreten sind.

$\mathrm{DaB}$ diese verschiedenen Intelligenzstörungen und spastischen Lähmungserscheinungen in der Mehrzahl der Fälle tatsächlich auf Geburtstraumen zurückgeführt werden sollten, scheint oberflächlich betrachtet etwas befremdend. Denn erstens nimmt man im allgemeinen an, daß die Geburt um so leichter ist, je kleiner das Kind. Wir müssen aber hier zwei Momente auseinander halten; das was für die Mutter leicht ist, das heißt eine in wenigen Minuten vor sich gehende Geburt, kann geradezu als eine schwere für das Kind betrachtet werden, denn bei starker Wehentätigkeit und bei schnell erfolgender Geburt kann doch der Kopf des Kindes sich nicht so gut an die Geburtswege adaptieren wie bei einer, die etwas langsamer vorwärts schreitet. Infolge der starken Wehen können sehr leicht auf einmal die Schädel- und Gehirngefäße zu stark mit Blut gefüllt werden. Die Gefäße sind nun aber bekanntlich bei Frühgeburten je kleiner, um so zerreißlicher, da die elastischen Fasern in der Gefäßwand bei den kleineren Frühgeburten noch sehr schwach entwickelt sind.

Dieser ätiologischen Erklärung könnte der Einwand entgegengehalten werden, daB die spastischen Erscheinungen kaum je gleich nach der Geburt nachzuweisen sind, was der Fall sein müßte, falls sie auf geburtstraumatische Gehirnresp. Rückenmarksblutungen zurückzuführen wären. Die Sache ist aber so, daß nach den bekannten Untersuchungen von Soltmans, Westphal u. a. die Gehirnrinde auch noch bei ausgetragenen Neugeborenen, geschweige denn bei Frühgeburten so unempfindlich ist, daß dieselbe nicht einmal auf direkte elektrische Reize mit Zuckungen oder dergleichen reagiert. So ist es auch bei ausgedehnteren Gehirnblutungen. Häufig sieht man bei Frühgeburten die ganze Konvexität des Gehirns mit dicken Blutgerinnseln überzogen, ohne daß diese Kinder irgendwelche deutlicheren cerebralen Krämpfe, Spasmen usw. gezeigt hätten. So entsteht auch der LitTLEsche Symptomenkomplex erst allmählich, nachdem die Blutungen resorbiert oder verschwunden sind, eventuell Narbengewebe hinterlassen haben und nachdem die Gehirnrinde mit der Zeit für verschiedene Reize empfindlicher geworden ist.

In diesem Zusammenhang muß auch noch erwähnt werden, $\mathrm{daB}$ sogenannte funktionelle oder spasmophile Krämpfe bei den Frühgeborenen besonders häufig auftreten. Hierbei muß man immer daran denken, daß bei diesen die Häufigkeit der Spasmophilie auch irgendwie im Zùsammenhang mit den geburtstraumatischen Gehirnschädigungen sein könnte. Hierauf deuten auch diejenigen Befunde, die wir bei unseren Nachforschungen gemacht haben. Zirka $40 \%$ derjenigen Frühgeburten, die sogenannte spasmophile Krämpfe im I. bis 2. Lebensjahre gehabt hatten, zeigten auch im späteren Leben verschiedenartige cerebrale Defekte, vor allem Intelligenzstörungen.

Hiermit wollen wir keineswegs leugnen, daß vermutlich verschiedene andere spätere, extrauterine, exogene Schädigungen, sei es alimentärer oder infektiöser Art, als auslösendes Moment beim Zustandekommen der spasmophilen Krämpfe die Hauptrolle spielen. Jedem Pädiater ist doch bekannt, wie schwer es ist, kleine Frühgeburten mit künstlicher Ernährung von Geburt an aufzuziehen, und gerade bei den Frühgeburten sieht man häufig, daß bald nach Beginn der künstlichen Ernährung die spasmophilen Krämpfe prompt einsetzen.

Von weiteren pathologischen Eigentümlichkeiten bei den Frühgeburten verdient hier die sogenannte Frühgeburtenanämie genauer besprochen $z u$ werden. Jede Frühgeburt zeigt bei der Geburt einen auffallend hohen Hämoglobingehalt (ca. I20 Sahli) und eine vermehrte Anzahl von roten Blutkörperchen $(6-7$ Millionen). Im extrauterinen Leben 
leiden aber scheinbar durch verschiedene exogene Ursachen die hämatopoetischen Organe, so daß trotz dieses Oberschusses an Hämoglobin und an roten Blutkörperchen bei der Geburt die frühgeborenen Kinder binnen kurzem anämisch werden. Am Ende des ersten Lebensmonats ist der Hämoglobingehalt nur noch 7o-8o Sahli und in den folgenden Lebensmonaten geht derselbe noch sukzessiv zurück, so daß die kleinsten Frühgeburten häufig im 4. bis 6 . Lebensmonat nur Werte von $40-50$ Sahli aufweisen.

Vom 6. Monat an fängt aber der Hämoglobingehalt wieder an zu steigen. Aber eine gewisse Anämie ist doch bei der Mehrzah1 der kleinsten Frühgeburten in den ersten Lebensjahren nachzuweisen. Diese Anämie ist bei den kleineren Frühgeburten eine unvermeidliche Erscheinung. Erkranken sie dann noch dazu an Ernährungsstörungen oder wiederholter Grippe, so wird die Anämie noch schwerer und hartnäckiger und die Kinder erholen sich bedeutend langsamer davon.

Die Frühgeburtenanämie zeigt in der Hauptsache das Bild der aplastischen Anämie. Die Anzahl der roten Blutkörperchen ist herabgesetzt, wenn auch nicht so viel, wie der Hämoglobingehalt. Therapeutisch hat sich hierbei Ferr. carb. sachar. $3 \times 0,2-0,3$ gut bewährt. Bei vorwiegender Milchernäbrung bekommen nämlich die Kinder viel zu wenig Eisen, um ihren Bedarf zu decken.

Hand in Hand mit der Frühgeburtenanämie geht auch die Entwickelung der Frühgeburtenrachitis. Die Entwicklung der Knochen ist überhaupt bei den kleineren Frühgeborenen bei der Geburt relativ mangelhaft. Z. B. besitzen die Kinder, die mit einem Geburtsgewicht unter $1600 \mathrm{~g}$ und weniger als $43 \mathrm{~cm}$ Länge geboren werden, noch keinen Knochenkern in der unteren Femurepiphyse. Grenau wie die verschiedenen Schädigungen, die das extrauterine Leben mit sich bringt, die Tätigkeit der hämatopoetischen Organe allmählich lähmen, genau so fängt auch das normale Knochenwachstum und der normale Kalkansatz bei den Frühgeburten bald nach der Geburt an zu leiden und gestört zu werden.

In Röntgenbildern können wir bereits bei einige Wochen alten Frühgeburten eine Rarefizierung der Knochenstruktur und Unregelmäßigkeiten in den Epiphysenlinien nachweisen, und klinisch kann man auch oft bereits bei 1-2 Monate alten Frühgeburten durch Weichwerden des Hinterkoptes (Craniotabes) das Auftreten der Rachitis feststellen. Gerade am Kopf können wir auch in den folgenden Monaten am deutlichsten die rachitischen Veränderungen sehen. Gleichzeitig mit dem Weichwerden des Hinterkopfes können nämlich dieTnbera frontalia und parietalia höckerartige Gebilde aufweisen.

Eigentümlicherweise treten an den Extremitäten bei den Frühgeburten in den ersten Jahren keine solche schweren Epiphysenauftreibungen und Verkrümmungen der Extremitäten auf, wie wir bei den dickeren ausgetragenen Kindern kennen. Dies hängt wohl damit zusammen, daß die Frühgeburten nur ausnahmsweise bereits im ersten Jahre ihre Extremitäten einer stärkeren Belastung aussetzen. Im allgemeinen scheinen ja die Verkrümmungen und Deformationen der Extremitäten als Folgeerscheinungen von mechanischer Ubberlastung oder intensiveren Bewegungen zu sein.

Bei der Therapie der Frühgeburtenrachitis hat sich am besten eine so frühzeitig wie möglich (bereits im $4 .-5$. Monat) einsetzende Beikosternährung (Kartoffelbrei und andere Gemüsearten) zusammen mit Kalk (Calc. phosphor. tribasic. 0,5 2-3 mal) bewährt. Die Frühgeburtenrachitis scheint ihrerseits auch einen verzögernden Einflu $\beta$ auf das Wachstum in den ersten Lebensjahren $z u$ haben, denn im allgemeinen bleiben die Frühgeburten in den ersten 3-4 Lebensjahren deutlich im Vergleich $z u$ gleichaltrigen ausgetragenen Kindern zurück. Späterhin gleicht sich aber der Unterschied aus und auch die kleinsten Frühgeburten können sich allmählich nicht nur, was die geistigen Fähigkeiten, sondern auch was die Körpergröße betrifft, zu ebenso vollwertigen Menschen entwickeln, wie irgendein anderes ausgetragenes Kind.

Wenn wir auch nach alledem bei den Frühgeburten vor allem mit einer großen Sterblichkeit und auch mit gehäuftem Auftreten von verschiedenen krankhaften Zuständen in den ersten Lebensjahren rechnen müssen, so können wir doch zu unserer Freude durch sorgsame Pflege auch aus den allerkleinsten Frühgeburten in vielen Fällen durchaus normale Menschen heranwachsen sehen.

\section{DIE HEILENDEN UND SCHÄDIGENDEN WIRKUNGEN DES SALVARSANS ${ }^{1}$ ).}

Von

Prof. Dr. J. JaDassohn (Breslau).

Direktor der Klinik für Syphilis u. Hautkrankh. d. Univ. Breslau.

\section{(SchluB.)}

Bei den mit Salvarsan in Beziehung: stehenden Lebererkrankungen unterscheiden wir bekanntlich den Früh- und den Spätikterus und die akute gelbe Leberatrophie. Die ersten beiden verlaufen meistenteils günstig, seltener halten sie sich längere Zeit und führen zu stärkerer Schädigung des Allgemeinbefindens. Beim Frühikterus hat man an eine Provokationswirkung gedacht, beim Spätikterus an ein „Hepatorezidiv". Er soll auf Salvarsanbehandlung gut zurückgehen. Darüber habe ich keine eigenen Erfahrungen. Dagegen kann ich in Obereinstimmung mit vielen anderen berichten, daß er ohne spezifische und unter Quecksilberbehandlung meist relativ leicht heilt. Die Behandlung ist nach allgemeinmedizinischen Prinzipien zu leiten (energische Durchspülungen). Trotz des Gedankens an eine syphilitische Komponente scheint mir eine weitere Zufuhr von Salvarsan beim Frühikterus, ein Wiederbeginn damit beim Spätikterus, wenigstens vorläufig noch nicht indiziert.

Nur in vereinzelten Fällen entwickelt sich aus dem Ikterus, in anderen entsteht ohne ihn die akute gelbe Leberatrophie. Diese Erkrankung ist, wie unzweifelhaft auch der Ikterus, in den letzten Jahren, wenigstens an manchen Orten, unabhängig von Syphilis und Salvarsan häufiger geworden. Es geht aber nach dem in der Literatur vorliegenden Material meines Erachtens nicht mehr an, ihren Zusammenhang mit der Salvarsanbehandlung zu leugnen, auch wenn man an die Bedeutung der Syphilis und der veränderten Ernährung oder einen "Genius hepaticus" denkt.

AuBer durch die Beachtung auch der leichtesten ikterischen Symptome, durch Urin- und Temperaturkontrolle, durch sorgfältige anamnestische Feststellung der früheren Gesundheit der Leber wird man zur Vermeidung dieser Krankheit nichts beitragen können. Wenn auch durch die Erfahrungen der letzten Jahre ihre Prognose nicht mehr so absolut ungünstig ist, wie man gemeint hat, so wird doch die Behandlung nicht gerade sehr viel zu ihrer Heilung tun. Neben der Durchspülung des Organismus wird man der Erhaltung des Kräftezustandes sein Hauptaugenmerk zuwenden müssen.

Endlich die ,Encephalitis haemorrhagica" und die außerordentlich viel selteneren, wahrscheinlich analogen Veränderungen im Rückenmark. Auch sie sind in ihrer Pathogenese nicht aufgeklärt, und zu ihrer Vermeidung können wir außer den allgemeinen VorsichtsmaBregeln kaum etwas wesentliches tun. Bei Fieber und Kopfschmerzen im Verlauf der Behandlung werden wir an die Möglichkeit denken müssen, daß sie Vorboten einer so schweren Erkrankung sein können, die Salvarsanzufuhr aussetzen und nur nach völligem Rückgang wieder beginnen (Lumbalpunktion, evtl. $\mathrm{Hg}$ - und Jodbehandlung). Bildet sich die Encephalitis aus, so geschieht das meist so plötzlich, daß wir sofort mit unserem ganzen therapeutischen Rüstzeug vorgehen müssen: Aderlaß und Infusionen von Kochsalzlösung, Lumbalpunktion, vor allem aber Adrenalininjektionen. $\mathrm{Ob}$ von dem ultimum Refugium, der Trepanation, schon erfolgreich Gebrauch gemacht worden ist, ist mir nicht bekannt. Dagegen gibt es doch eine nicht ganz kleine Anzahl geheilter Fälle. Ich selbst habe deren 3 und keinen Todesfall beobachtet (einen nicht diagnostizierten vor dem Belsanntwerden dieser schwersten Schädigung ohne Behandlung, 2 mit Adrenalin). Seit einer Anzahl von Jahren habe ich überhaupt einen solchen Fall nicht mehr gesehen. 